\title{
使用局部支撑径向基函数的隐式曲线曲面几何迭代算法
}

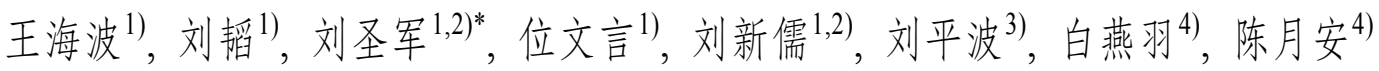 \\ 1) (中南大学数学与统计学院 长沙 410083) \\ 2) (中南大学工程建模与科学计算研究所 长沙 410083) \\ 3) (中南林业科技大学计算机与信息工程学院 长沙 410004) \\ 4) (中国航发南方工业有限公司 株洲 412000) \\ (shjliu.cg@csu.edu.cn)
}

\begin{abstract}
摘 要：由散乱数据稳定重构曲线曲面，在变分拟插值方法的基础之上，提出了使用局部支撑径向基函数的隐式几 何迭代算法. 首先, 根据给定数据点的法向构造隐式函数的非零约束, 构造计算隐函数系数的迭代格式, 并讨论其收 玫性; 然后，在此基础上引人加速因子，对隐式迭代算法进行加速，同时讨论了加速算法的收敛性；最后，为了降低 迭代过程空间和时间的复杂度，给出了一种加速算法的改进版本. 数值实验表明，使用局部支撑径向基函数的隐式 几何迭代算法对曲线曲面重构是有效的，并对部分信息缺失、非均匀分布、带噪声采样数据的重构也达到了较好的 效果，且实现简单，易于并行.
\end{abstract}

关键词：隐式几何迭代算法; 加速因子; 局部支撑径向基函数; 曲线曲面重构; 拟插值

中图法分类号: TP391.41 DOI: 10.3724/SP.J.1089.2021.18807

\section{Implicit Progressive-Iterative Algorithm of Curves and Surfaces with Compactly Supported Radial Basis Functions}

\author{
Wang Haibo ${ }^{1)}$, Liu Tao ${ }^{1)}$, Liu Shengjun ${ }^{1,2)^{*}}$, Wei Wenyan ${ }^{1)}$, Liu Xinru ${ }^{1,2)}$, Liu Pingbo ${ }^{3)}$, Bai Yanyu ${ }^{4)}$, and \\ Chen Yue'an ${ }^{4)}$ \\ 1) (School of Mathematics and Statistics, Central South University, Changsha 410083) \\ ${ }^{2)}$ (Institute of Engineering Modeling and Scientific Computing, Central South University, Changsha 410083) \\ 3) (School of Computer and Information Engineering, Central South University of Forestry and Technology, Changsha 410004) \\ 4) (AECC South Industry Company Limited, Zhuzhou 412000)
}

\begin{abstract}
To reconstruct curves and surfaces robustly from scattered data, an implicit progressive-iterative algorithm with compactly supported radial basis functions based on the variational quasi-interpolation method is proposed. Firstly, the non-zero constraint of the implicit function is constructed using normal vectors at the given points, an iterative scheme for calculating coefficients of the implicit function is developed and its convergence is discussed. Secondly, by introducing an acceleration factor, the implicit progressive-iteration algorithm is sped up, and the convergence is analyzed. Finally, the accelerated algorithm is modified to decrease the space and time complexity. Numerical experiments show that the algorithm is effective for curve and surface reconstruction, and it also achieves good results for reconstructing from data with missing samples, non-uniform distribution, and
\end{abstract}

收稿日期：2020-11-22; 修回日期：2021-03-11. 基金项目：国家自然科学基金(61602524); 湖南省重点研发计划(2017NK2383); 中南大学研究生自主探索创新项目(2021zzts0474). 王海波(1997-), 男, 硕士研究生, 主要研究方向为计算机辅助几何设计; 刘韬 (1995-), 男, 硕士研究生, 主要研究方向为计算机图形学、计算机辅助设计; 刘圣军(1979-), 男, 博士, 教授, 博士生导师, CCF 会 员, 论文通讯作者, 主要研究方向为几何造型、数字几何处理、数据分析与智能算法; 位文言 (1993-), 女, 硕士研究生, 主要研究方 向为计算机辅助几何设计; 刘新儒(1982-), 男, 博士, 副教授, 硕士生导师, 主要研究方向为几何造型、数值建模、数据分析与智能 算法; 刘平波(1982一), 女, 硕士, 讲师, 主要研究方向为计算机信息技术、3S 技术与应用; 白燕羽(1987一), 男, 硕士, 工程师, 主要 研究方向为控制系统、科学计算、工程建模; 陈月安(1982-), 男, 硕士, 工程师, 主要研究方向为控制系统、科学计算、工程建模. 
noises. Moreover, it is simple to implement and easy to process in parallel.

Key words: implicit progressive-iterative approximation algorithm; acceleration factor; compactly supported radial basis function; reconstruction of curve and surface; quasi-interpolation

\section{1 相关工作}

近年来，随着三维扫描技术的广泛使用，利用 采样点进行曲面重构越来越重要, 已成为一个研 究热点, 吸引了众多研究学者, 产生了许多重构技

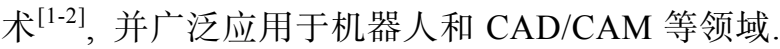
而径向基函数(radical basis function, RBF)具有计 算简单、可处理非均匀采样点的特点, 备受研究学 者的青睐，基于 $\mathrm{RBF}$ 的重构方法成为目前的主流 方法之一.

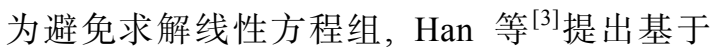
$\mathrm{RBF}$ 的拟插值技术, 并给出了形状参数的取值. $\mathrm{Liu}$ 等 ${ }^{[4]}$ 将数据推广到三维散乱点, 取得了较好的 重构效果，但是局部二次拟合仍需要求解小型线 性方程组. Pan 等 ${ }^{[5]}$ 提出一种基于大量三维散乱数 据点及其相关的法向量重构曲面的方法. Liu 等 ${ }^{[6]}$ 在其基础上加以改进，提出了基于多层拟插值的 Hermite 变分隐式曲面重构方法, 直接利用法向量 构造非零约束, 分层选取支撑半径, 实现曲面重 构. 但是支撑半径的大小对重建效果影响较大, 尤 其是对于非均匀分布的点云数据, 支撑半径太小, 重构结果会出现空洞; 而支撑半径太大, 则会导致 细节丢失。

几何迭代法(geometric iterative method, GIM) 又称为渐进迭代逼近法 (progressive-iterative approximation, PIA), 是一类具有明显几何意义的曲 线曲面插值(拟合)的迭代算法，其操作简单，易于 并行. 该算法最初起源于齐东旭等 ${ }^{[7]}$ 提出的均匀三 次 B 样条曲线的“盈亏修正算法”. 随后 de Boor ${ }^{[8]}$ 证明了其收玫性. 2004 年, Lin 等 ${ }^{[9]}$ 证明了非均匀三 次 B 样条函数的“盈亏修正”也是收玫的. 进一步, Lin 等 ${ }^{[10]}$ 证明了归一化全正基曲线曲面相应迭代 算法的收玫性，并将这一算法命名为 PIA，从此开 始了 PIA 和 GIM 的系列研究. 而后, $\operatorname{Lin}^{[11]}$ 指出, 该算法具有局部性质，并提出了局部 PIA 算法 (local progressive-iterative approximation, LPIA). 针对大规模数据, 提出了 PIA 推广格式(extended progressive-iterative approximation, EPIA)和最小二 乘 PIA (least square progressive-iterative approxi-

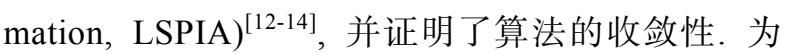

了加速 $\mathrm{PIA}, \mathrm{Lu}^{[15]}$ 和张莉等 ${ }^{[16]}$ 通过引人加速因子, 分别给出了加权 PIA 算法 (weighted progressive-iterative approximation, WPIA), 提高了算法效 率. 但是文献[15]中的最优权重需要计算配置矩阵 的最小特征值, 文献[16]中权系数中的参数较难确 定. 除参数形式的 PIA 外, Hamza 等 ${ }^{[17]}$ 提出了基于 B 样条的隐式曲线曲面迭代算法(implicit progressive-iterative approximation, IPIA), 并证明了收敛 性，同时给出了最优加速因子和实用加速因子.

文献[17]使用的基函数为 $\mathrm{B}$ 样条，而本文使用 计算更加简便的局部支撑 RBF，构造以拟插值结 果为初值、基于局部支撑 RBF 的隐式 PIA 格式. 为 了提高迭代收玫速度，设计最优权重隐式 PIA 加 速算法. 对于采样点数量较大的数据, 为了进一步 提高计算速度并降低空间复杂度，采用估计最小 特征值方法计算最优权重, 实现更快速的隐式 PIA 算法.

\section{2 隐式 PIA}

\section{1 隐式 PIA 算法介绍}

给定曲线曲面上一个数据点列 $\boldsymbol{P}=\left\{\boldsymbol{p}_{1}\right.$, $\left.\boldsymbol{p}_{2}, \cdots, \boldsymbol{p}_{n}\right\}\left(\boldsymbol{p}_{i} \in \mathbb{R}^{2}\right.$ 或 $\left.\mathbb{R}^{3}, i=1,2, \cdots, n\right)$ 以及该点列 所对应的法向量 $N=\left\{n_{1}, n_{2}, \cdots, n_{n}\right\} \quad\left(n_{i} \in \mathbb{R}^{2}\right.$ 或 $\left.\mathbb{R}^{3}, i=1,2, \cdots, n\right)$, 其中法向量的方向保持一致，同 时向外或向内, 则该曲线曲面对应于隐式函数 $f$ 标量场中的零值面，即 $f\left(\boldsymbol{p}_{i}\right)=0, i=1,2, \cdots, n$. 根 据文献[6]构造隐式函数, 其表达式为

$$
f(\boldsymbol{x})=\sum_{i=1}^{n} c_{i} \varphi_{i}(\boldsymbol{x})+\sum_{i=1}^{n}\left\langle\boldsymbol{n}_{i}, \nabla \varphi_{i}(\boldsymbol{x})\right\rangle=0
$$

其中, $\boldsymbol{x}$ 表示数据点; $c_{i}$ 表示由插值约束决定的 未知系数; $\langle\cdot$,$\rangle 表示 2$ 个向量的点积; $\nabla \varphi_{i}(\boldsymbol{x})$ 表 示 $\varphi_{i}(\boldsymbol{x})$ 的梯度； $\varphi_{i}(\boldsymbol{x})$ 表示归一化的 RBF，其表 达式为

$$
\varphi_{i}(\boldsymbol{x})=\frac{\phi_{\rho}\left(\left\|\boldsymbol{x}-\boldsymbol{p}_{i}\right\|\right)}{\sum_{j=1}^{n} \phi_{\rho}\left(\left\|\boldsymbol{x}-\boldsymbol{p}_{j}\right\|\right)} .
$$

其中, $\left\|\boldsymbol{x}-\boldsymbol{p}_{i}\right\|$ 表示点 $\boldsymbol{x}$ 到 $\boldsymbol{p}_{i}$ 的欧几里得度量. 本 
文使用 Wendland 提出的局部支撑 RBF(compactly supported radial basis function, CSRBF $)^{[2]}$ 作为归一化 RBF 中的核函数, 即

$$
\begin{gathered}
\phi_{\rho}(r)=\phi\left(\frac{r}{\rho}\right), \\
\phi(r)=\left\{\begin{array}{ll}
(1-r)^{4}(4 r+1), & r \in[0,1] \\
0, & \text { 其他 }
\end{array} .\right.
\end{gathered}
$$

其中, $\rho$ 表示支撑半径; $r=\|\boldsymbol{p}-\boldsymbol{q}\|$ 表示数据点 $\boldsymbol{p}$ 与 RBF 中心 $\boldsymbol{q}$ 的欧几里得距离.

在进行迭代前, 首先令

$$
c_{i}^{(0)}=-\sum_{j=1}^{n}\left\langle\boldsymbol{n}_{j}, \nabla \varphi_{j}\left(\boldsymbol{p}_{i}\right)\right\rangle_{=}^{\Delta} d_{i}, i=1,2, \cdots, n
$$

其中, $d_{i}$ 为由 $g(\boldsymbol{x})=\sum_{i=1}^{n} c_{i} \varphi_{i}(\boldsymbol{x})$ 定义的距离场中的 距离值，表示空间中点 $\boldsymbol{p}_{j}$ 在每个点 RBF 中心点 $\boldsymbol{p}_{i}$ 所定义场中的梯度与 $\boldsymbol{p}_{j}$ 的法向上投影之和.

将式(2)代人式(1), 得到初始曲线曲面

$$
\begin{gathered}
f^{(0)}(\boldsymbol{x})=\sum_{i=1}^{n}\left(-\sum_{j=1}^{n}\left\langle\boldsymbol{n}_{j}, \nabla \varphi_{j}\left(\boldsymbol{p}_{i}\right)\right\rangle\right) \varphi_{i}(\boldsymbol{x})+ \\
\sum_{i=1}^{n}\left\langle\boldsymbol{n}_{i}, \nabla \varphi_{i}(\boldsymbol{x})\right\rangle=0
\end{gathered}
$$

算法 1. 隐式 PIA 算法.

输人. 一个数据点列 $\boldsymbol{P}=\left\{\boldsymbol{p}_{1}, \boldsymbol{p}_{2}, \cdots, \boldsymbol{p}_{n}\right\}$ 及其 相应的法向量 $\boldsymbol{N}=\left\{\boldsymbol{n}_{1}, \boldsymbol{n}_{2}, \cdots, \boldsymbol{n}_{n}\right\}$, 容许误差精度 $\varepsilon$, 最大迭代次数 $M$.

输出. 迭代曲线曲面.

Step1. 根据式(3)构造初始曲线曲面.

Step2. 令 $g^{(0)}(x)=\sum_{j=1}^{n} c_{j}^{(0)} \varphi_{j}(x)$, 计算差 $4^{(0)}=d_{i}-$ $g^{(0)}\left(\boldsymbol{p}_{i}\right)$.

Step3. 计算新的参数 $c_{i}^{(1)}=c_{i}^{(0)}+4_{i}^{(0)}$.

Step4. 生成新的曲线曲面

$$
f^{(1)}(\boldsymbol{x})=\sum_{i=1}^{n} c_{i}^{(1)} \varphi_{i}(\boldsymbol{x})+\sum_{i=1}^{n}\left\langle\boldsymbol{n}_{i}, \nabla \varphi_{i}(\boldsymbol{x})\right\rangle=0 .
$$

Step5. 重复 Step2 Step4, 第 $k$ 次迭代为

$$
\begin{aligned}
& g^{(k)}(\boldsymbol{x})=\sum_{j=1}^{n} c_{j}^{(k)} \varphi_{j}(\boldsymbol{x}) ; \\
& 4_{i}^{(k)}=d_{i}-g^{(k)}\left(\boldsymbol{p}_{i}\right) ; \\
& c_{i}^{(k+1)}=c_{i}^{(k)}+4^{(k)} ; \\
& f^{(k+1)}(\boldsymbol{x})=\sum_{i=1}^{n} c_{i}^{(k+1)} \varphi_{i}(\boldsymbol{x})+\sum_{i=1}^{n}\left\langle\boldsymbol{n}_{i}, \nabla \varphi_{i}(\boldsymbol{x})\right\rangle=0 .
\end{aligned}
$$

Step6. 当误差达到预先给定的容许误差精度 $\varepsilon$ 或迭 代次数达到预先给定的最大迭代次数 $M$ 时, 算法终止.

\section{2 隐式 PIA 算法收敛性分析}

算法 1 迭代过后得到曲线曲面序列 $\left\{f^{(0)}(\boldsymbol{x})\right.$, $\left.f^{(1)}(\boldsymbol{x}), \cdots, f^{(k)}(\boldsymbol{x}), \cdots\right\}$, 当 $\lim _{k \rightarrow \infty} f^{(k)}\left(\boldsymbol{p}_{i}\right)=0$ 成立, 即 该曲线曲面序列收玫到给定点时, 则称该方法具 有 PIA 性质.

实际上, 由算法 1 可知, 第 $k+1$ 次迭代的差可 表示为

$4_{i}^{(k+1)}=d_{i}^{(0)}-g^{(k+1)}\left(\boldsymbol{p}_{i}\right)=4_{i}^{(k)}+g^{(k)}\left(\boldsymbol{p}_{i}\right)-g^{(k+1)}\left(\boldsymbol{p}_{i}\right)$. 而 $g^{(k)}(\boldsymbol{x})=\sum_{i=1}^{n} c_{i}^{(k)} \varphi_{i}(\boldsymbol{x}), g^{(k+1)}(\boldsymbol{x})=\sum_{i=1}^{n} c_{i}^{(k+1)} \varphi_{i}(\boldsymbol{x})$. 故有

$$
\begin{aligned}
& \Delta_{i}^{(k+1)}=\Delta_{i}^{(k)}+\sum_{j=1}^{n} c_{j}^{(k)} \varphi_{j}\left(\boldsymbol{p}_{i}\right)-\sum_{j=1}^{n} c_{j}^{(k+1)} \varphi_{j}\left(\boldsymbol{p}_{i}\right)= \\
& \Delta_{i}^{(k)}-\sum_{j=1}^{n}\left(c_{j}^{(k+1)}-c_{j}^{(k)}\right) \varphi_{j}\left(\boldsymbol{p}_{i}\right)= \\
& \Delta_{i}^{(k)}-\sum_{j=1}^{n} \Delta_{j}^{(k)} \varphi_{j}\left(\boldsymbol{p}_{i}\right)
\end{aligned}
$$

记 $\Delta^{(k)}=\left[\Delta_{1}^{(k)}, \Delta_{2}^{(k)}, \cdots, \Delta_{n}^{(k)}\right]^{\mathrm{T}}$, 则式(4)写成矩阵形 式为 $\boldsymbol{\Delta}^{(k+1)}=(\boldsymbol{I}-\boldsymbol{B}) \boldsymbol{\Delta}^{(k)}$. 其中, $\boldsymbol{I}$ 为 $n$ 阶单位矩阵; $\boldsymbol{B}$ 为将点列 $\boldsymbol{P}=\left\{\boldsymbol{p}_{1}, \boldsymbol{p}_{2}, \cdots, \boldsymbol{p}_{n}\right\}$ 代人基函数 $\varphi_{i}(\boldsymbol{x})$ $(i=1,2, \cdots, n)$ 中后组成的 $n$ 阶归一化配置矩阵, 即

$$
\boldsymbol{B}=\left[\begin{array}{cccc}
\varphi_{1}\left(\boldsymbol{p}_{1}\right) & \varphi_{2}\left(\boldsymbol{p}_{1}\right) & \cdots & \varphi_{n}\left(\boldsymbol{p}_{1}\right) \\
\varphi_{1}\left(\boldsymbol{p}_{2}\right) & \varphi_{2}\left(\boldsymbol{p}_{2}\right) & \cdots & \varphi_{n}\left(\boldsymbol{p}_{2}\right) \\
\vdots & \vdots & \ddots & \vdots \\
\varphi_{1}\left(\boldsymbol{p}_{n}\right) & \varphi_{2}\left(\boldsymbol{p}_{n}\right) & \cdots & \varphi_{n}\left(\boldsymbol{p}_{n}\right)
\end{array}\right] .
$$

为证明该迭代算法的收玫性, 首先给出一个 引理和定义.

引理 $\mathbf{1}^{[18]}$. 矩阵 $\boldsymbol{A}$ 的任意特征值 $\lambda$ 的模都不超 过矩阵 $\boldsymbol{A}$ (在任意矩阵范数 $\|\cdot\|$ 定义下) 的范数 $\|\boldsymbol{A}\|$, 即 $|\lambda| \leqslant\|A\|$.

定义 $\mathbf{1}^{[18]}$. 矩阵的谱半径 $\rho(\boldsymbol{B})$ 是 $n$ 阶矩阵 $\boldsymbol{B}$ 特征值绝对值集合 $\left\{\left|\lambda_{i}(\boldsymbol{B})\right|, i=1,2, \cdots, n\right\}$ 的上确界.

定理 1. 算法 1 具有 PIA 性质.

证明. 由引理 1 和定义 1 可得, 配置矩阵 $\boldsymbol{B}$ 的 谱半径满足 $\rho(\boldsymbol{B}) \leqslant\|\boldsymbol{B}\|_{\infty}=\max _{1 \leqslant i \leqslant n} \sum_{j=1}^{n}\left|\varphi_{i}\left(\boldsymbol{p}_{j}\right)\right|$.

由于 RBF 已经进行归一化处理, 故配置矩阵 $\boldsymbol{B}$ 的特征值 $\lambda_{i}(\boldsymbol{B})(i=1,2, \cdots, n)$ 满足 $\left|\lambda_{i}(\boldsymbol{B})\right| \leqslant 1$.

另外, 有 $\boldsymbol{B}=\boldsymbol{C D}$, 其中, 


$$
\begin{aligned}
& \boldsymbol{C}=\left[\begin{array}{rrrrrr}
1 / \sum_{j=1}^{n} \eta_{1 j} & & 0 & \cdots & & 0 \\
0 & 1 / \sum_{j=1}^{n} \eta_{2 j} & \cdots & & 0 \\
\vdots & & \vdots & \ddots & & \vdots \\
0 & & 0 & \cdots & 1 / \sum_{j=1}^{n} \eta_{n j}
\end{array}\right] ; \\
& \boldsymbol{D}=\left[\begin{array}{cccc}
\eta_{11} & \eta_{12} & \cdots & \eta_{1 n} \\
\eta_{21} & \eta_{22} & \cdots & \eta_{2 n} \\
\vdots & \vdots & \ddots & \vdots \\
\eta_{n 1} & \eta_{n 2} & \cdots & \eta_{n n}
\end{array}\right] \\
& \eta_{i j}=\phi_{\rho}\left(\left\|\boldsymbol{p}_{i}-\boldsymbol{p}_{j}\right\|\right) \text {. }
\end{aligned}
$$

$C$ 为对角矩阵，且对角线元素均为正， $\boldsymbol{D}$ 为对称 正定矩阵 ${ }^{[2]}$. 由文献 [19] 可知, $\lambda_{i}(B)>0, i=1,2$, $\cdots, n$, 故有 $0<\lambda_{i}(\boldsymbol{B}) \leqslant 1, i=1,2, \cdots, n$. 则 $\boldsymbol{I}-\boldsymbol{B}$ 的 特征值满足 $0 \leqslant\left|\lambda_{i}(\boldsymbol{I}-\boldsymbol{B})\right|=1-\left|\lambda_{i}(\boldsymbol{B})\right|<1, i=1,2, \cdots$, $n ; \boldsymbol{I}-\boldsymbol{B}$ 的谱半径满足 $0 \leqslant \rho(\boldsymbol{I}-\boldsymbol{B})<1$. 故隐式 曲线曲面的 PIA 算法收敛, 算法 1 具有 PIA 性质.

证毕.

\section{3 隐式 PIA 加速算法}

\section{1 隐式 PIA 加速算法介绍}

在算法 1 的基础上，引人加速因子 $\omega$, 对算法 1 进行加速, 形成算法 2 .

算法 2. 隐式 PIA 加速算法.

输人. 一个数据点列 $\boldsymbol{P}=\left\{\boldsymbol{p}_{1}, \boldsymbol{p}_{2}, \cdots, \boldsymbol{p}_{n}\right\}$ 及其 相应的法向量 $\boldsymbol{N}=\left\{\boldsymbol{n}_{1}, \boldsymbol{n}_{2}, \cdots, \boldsymbol{n}_{n}\right\}$, 容许误差精度 $\varepsilon$, 最大迭代次数 $M$.

输出. 迭代曲线曲面.

Step1. 根据式(3)构造初始曲线曲面.

Step2. 令 $g^{(0)}(\boldsymbol{x})=\sum_{j=1}^{n} c_{j}^{(0)} \varphi_{j}(\boldsymbol{x})$, 计算差 $4^{(0)}=d_{i}-$ $g^{(0)}\left(\boldsymbol{p}_{i}\right)$.

Step3. 计算新的参数 $c_{i}^{(1)}=c_{i}^{(0)}+\omega \Delta_{i}^{(0)}$.

Step4. 生成新的曲线曲面

$f^{(1)}(\boldsymbol{x})=\sum_{i=1}^{n} c_{i}^{(1)} \varphi_{i}(\boldsymbol{x})+\sum_{i=1}^{n}\left\langle\boldsymbol{n}_{i}, \nabla \varphi_{i}(\boldsymbol{x})\right\rangle=0$.

Step5. 重复 Step2 Step4, 第 $k$ 次迭代为

$$
\begin{aligned}
& g^{(k)}(\boldsymbol{x})=\sum_{j=1}^{n} c_{j}^{(k)} \varphi_{j}(\boldsymbol{x}) ; \\
& 4_{i}^{(k)}=d_{i}-g^{(k)}\left(\boldsymbol{p}_{i}\right) ; \\
& c_{i}^{(k+1)}=c_{i}^{(k)}+\omega \Delta_{i}^{(k)} ; \\
& f^{(k+1)}(\boldsymbol{x})=\sum_{i=1}^{n} c_{i}^{(k+1)} \varphi_{i}(\boldsymbol{x})+\sum_{i=1}^{n}\left\langle\boldsymbol{n}_{i}, \nabla \varphi_{i}(\boldsymbol{x})\right\rangle=0 .
\end{aligned}
$$

Step6. 当误差达到预先给定的容许误差精度 $\varepsilon$ 或迭 代次数达到预先给定的最大迭代次数 $M$ 时, 算法终止.

\section{2 隐式 PIA 加速算法收敛性分析}

事实上, 由算法 2 可知, 第 $k+1$ 次迭代的差可 表示为

$$
\begin{aligned}
& \Delta_{i}^{(k+1)}=d_{i}-g^{(k+1)}\left(\boldsymbol{p}_{i}\right)=\Delta_{i}^{(k)}+g^{(k)}\left(\boldsymbol{p}_{i}\right)-g^{(k+1)}\left(\boldsymbol{p}_{i}\right) . \\
& \text { 而 } g^{(k)}(\boldsymbol{x})=\sum_{i=1}^{n} c_{i}^{(k)} \varphi_{i}(\boldsymbol{x}), g^{(k+1)}(\boldsymbol{x})=\sum_{i=1}^{n} c_{i}^{(k+1)} \varphi_{i}(\boldsymbol{x}) .
\end{aligned}
$$

故有

$$
\begin{aligned}
& \Delta_{i}^{(k+1)}=\Delta_{i}^{(k)}+\sum_{j=1}^{n} c_{j}^{(k)} \varphi_{j}\left(\boldsymbol{p}_{i}\right)-\sum_{j=1}^{n} c_{j}^{(k+1)} \varphi_{j}\left(\boldsymbol{p}_{i}\right)= \\
& \Delta_{i}^{(k)}-\sum_{j=1}^{n}\left(c_{j}^{(k+1)}-c_{j}^{(k)}\right) \varphi_{j}\left(\boldsymbol{p}_{i}\right)= \\
& \Delta_{i}^{(k)}-\omega \sum_{j=1}^{n} \Delta_{j}^{(k)} \varphi_{j}\left(\boldsymbol{p}_{i}\right)
\end{aligned}
$$

记 $\Delta^{(k)}=\left[\Delta_{1}^{(k)}, \Delta_{2}^{(k)}, \cdots, \Delta_{n}^{(k)}\right]^{\mathrm{T}}$, 则将式(5)写成矩阵 形式为 $\boldsymbol{\Delta}^{(k+1)}=(\boldsymbol{I}-\boldsymbol{\omega} \boldsymbol{B}) \boldsymbol{\Delta}^{(k)}$. 其中, $\boldsymbol{I}$ 为 $n$ 阶单位 矩阵; $\boldsymbol{B}$ 为 $n$ 阶归一化配置矩阵, 形式与第 2.2 节 中的 $\boldsymbol{B}$ 相同.

定理 2. 当 $0<\omega<2$ 时, 算法 2 具有 PIA 性质. 证明. 因为

$$
\rho(\boldsymbol{I}-\omega \boldsymbol{B})=\max \left\{|1-\omega|,\left|1-\omega \lambda_{\min }(\boldsymbol{B})\right|\right\} .
$$

其中, $\lambda_{\min }(\boldsymbol{B})$ 为 $\boldsymbol{B}$ 的最小特征值. 当 $0<\omega<2$ 时, 有 $0 \leqslant \rho(\boldsymbol{I}-\omega \boldsymbol{B})<1$ 成立，故算法 2 具有 PIA 性质.

证毕.

定理 3. 当 $\omega=\frac{2}{1+\lambda_{\text {min }}(\boldsymbol{B})}$ 时, 其中, $\lambda_{\text {min }}(\boldsymbol{B})$ 为 $\boldsymbol{B}$ 的最小特征值, 算法 2 的收敛速度最快.

证明. 算法 2 的收敛速度主要由 $\rho(\boldsymbol{I}-\omega \boldsymbol{B})$ 决 定, $\rho(\boldsymbol{I}-\omega \boldsymbol{B})$ 越小, 收玫越快. 故要证明定理 3, 只 需证明当 $\omega=\frac{2}{1+\lambda_{\text {min }}(\boldsymbol{B})}$ 时, $\rho(\boldsymbol{I}-\omega \boldsymbol{B})$ 最小即可.

当 $\omega=1$ 时, 有

$$
\rho(\boldsymbol{I}-\omega \boldsymbol{B})=\rho(\boldsymbol{I}-\boldsymbol{B})=1-\lambda_{\text {min }}(\boldsymbol{B}) .
$$

当 $0<\omega<1$ 时, 有

$$
\begin{gathered}
\rho(\boldsymbol{I}-\omega \boldsymbol{B})=\max \left\{|1-\omega|,\left|1-\omega \lambda_{\min }(\boldsymbol{B})\right|\right\}= \\
1-\omega \lambda_{\min }(\boldsymbol{B})>1-\lambda_{\min }(\boldsymbol{B}) .
\end{gathered}
$$

当 $1<\omega<2$ 时, 分为 2 种情况.

若 $\omega \lambda_{\text {min }}(\boldsymbol{B})>1$, 则有

$$
\begin{aligned}
& \rho(\boldsymbol{I}-\omega \boldsymbol{B})=\max \left\{|1-\omega|,\left|1-\omega \lambda_{\min }(\boldsymbol{B})\right|\right\}= \\
& \max \left\{\omega-1, \omega \lambda_{\min }(\boldsymbol{B})-1\right\} \geqslant \omega-1> \\
& \frac{1}{\lambda_{\text {min }}(\boldsymbol{B})}-1 \geqslant 1-\lambda_{\text {min }}(\boldsymbol{B}) .
\end{aligned}
$$


若 $\omega \lambda_{\text {min }}(\boldsymbol{B}) \leqslant 1$, 则有

$$
\begin{aligned}
& \rho(\boldsymbol{I}-\omega \boldsymbol{B})=\max \left\{|1-\omega|,\left|1-\omega \lambda_{\min }(\boldsymbol{B})\right|\right\}= \\
& \quad \max \left\{\omega-1,1-\omega \lambda_{\text {min }}(\boldsymbol{B})\right\}= \\
& \left\{\begin{array}{l}
1-\omega \lambda_{\text {min }}(\boldsymbol{B}), 1<\omega<\frac{2}{1+\lambda_{\text {min }}(\boldsymbol{B})} . \\
\omega-1, \frac{2}{1+\lambda_{\text {min }}(\boldsymbol{B})} \leqslant \omega<2
\end{array}\right.
\end{aligned}
$$

综上, 当 $\omega=\frac{2}{1+\lambda_{\text {min }}(\boldsymbol{B})}$ 时, $\rho(\boldsymbol{I}-\omega \boldsymbol{B})$ 取得最 小值 $\frac{1-\lambda_{\min }(\boldsymbol{B})}{1+\lambda_{\min }(\boldsymbol{B})}$.

证毕.

\section{4 实验结果与分析}

由于本文算法是以文献[6]中单层拟插值的结果 为初值进行迭代，以获得最后的重构结果，因此该 算法重构曲线曲面的精度比文献[6]更高, 但需要额 外的迭代时间. 另外, 文献[6]已经与一些经典的曲 面重构算法(如 MPU ${ }^{[20]}$, Poisson 重构算法 ${ }^{[21]}$ 等)进行 过比较, 在文中不再与这些方法比较. 采用曲线曲 面重构的实例，只与文献[6]中单层拟插值算法的结果 进行比较，以说明迭代算法的有效性和实用性. 本文 所有实验均在配置为 Intel(R) Core(TM)i5-9600K, $3.70 \mathrm{GHz}$ CPU 和 $8.0 \mathrm{~GB}$ 内存的计算机上进行.

本文所有的二维形状放缩到一个 $20 \times 20$ 的正
方形中, 所有的三维形状放缩到一个 $20 \times 20 \times 20$ 的 立方体中. 统计的重构结果最大几何误差为 $E\left(M, M^{\prime}\right)=\frac{D\left(M, M^{\prime}\right)}{D(M)}$. 其中, $D\left(M, M^{\prime}\right)$ 表示原始 模型 $M$ 与重构模型 $M^{\prime}$ 之间的最大距离; $D(M)$ 表 示原始模型 $M$ 的最小包围盒的对角线长度.

\section{1 算法有效性}

当选择合适的支撑半径时，单层拟插值算法 ${ }^{[6]}$ 基本能够满足需要, 但是当支撑半径偏大或偏小 时, 其效果却不尽人意, 可能导致细节特征丢失或 重构曲面有空洞. 而合适的支撑半径通常难以直 接选取到. 本文利用隐式 PIA 算法(算法 1 和算法 2)对图形初始重构形状进行修正, 即使选择的支 撑半径较大, 也能避免几何细节的丢失. 曲线重构 结果如图 1 图 3 所示, 曲面重构结果及其与拟插 值几何误差如图 4 图 6 所示. 不难发现, 经过隐式 几何迭代后, 重构结果保留了原始数据所拥有的 几何细节, 逼近误差更小, 如图 4c、图 5c、图 6c 和图 4d、图 5d、图 6d 所示.

表 1 列出了测试实例的统计信息, 包括模型点 的个数、支撑半径大小、重构结果最大逼近误差以 及不同算法的对应迭代时间和迭代次数. 从表 1 的 统计数据可以看出, 要达到相同的逼近误差, 经过 加速后的算法 2 收玫速度更快, 所需要的迭代次数 比算法 1 更少, 可以达到加速近 2 倍的效果. 当数

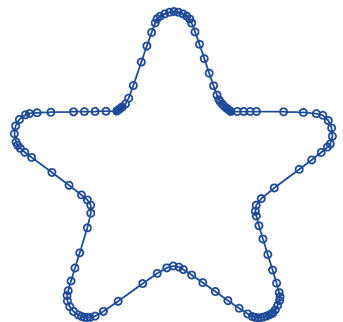

a. 原始采样点

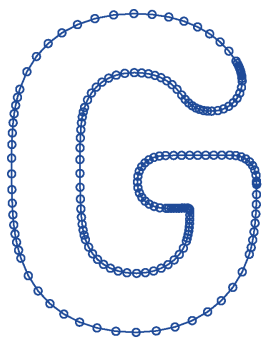

a. 原始采样点

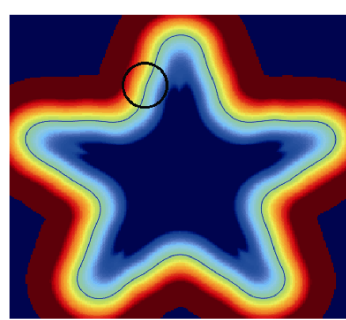

b. 拟插值结果 ${ }^{[6]}$

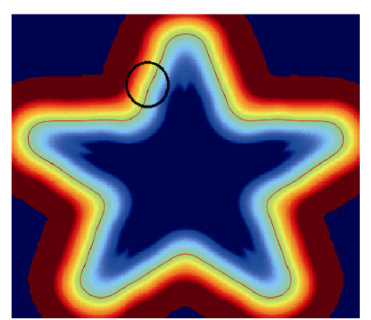

c. 迭代10次结果

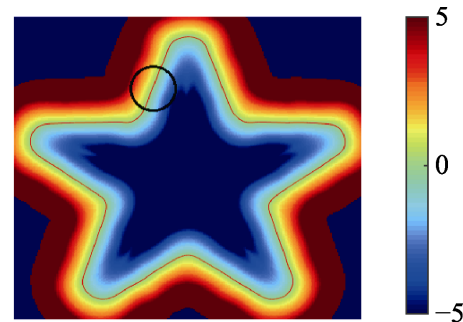

d. 迭代75次结果

图 1 五角星图形拟插值及隐式曲线几何迭代的重构结果

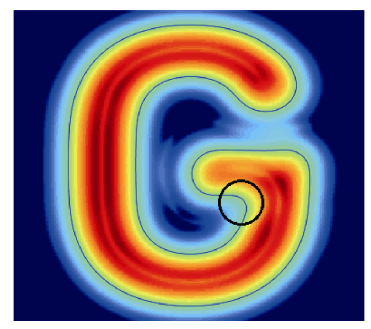

b. 拟插值结果 ${ }^{[6]}$

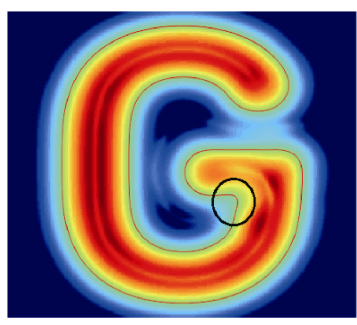

c. 迭代10次结果

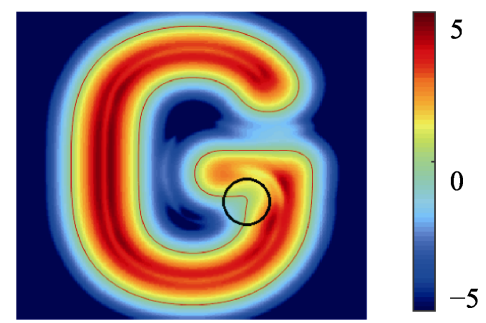

d. 迭代76次结果

图 2 字母 $\mathrm{G}$ 形状拟插值及隐式曲线几何迭代的重构结果 


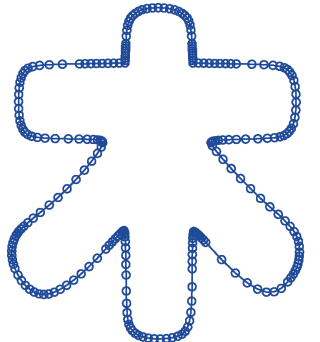

a. 原始采样点

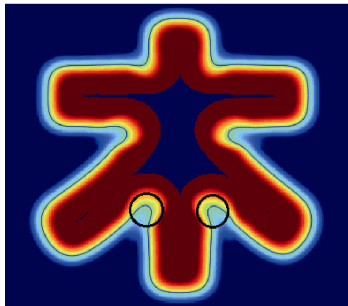

b. 拟插值结果 ${ }^{[6]}$

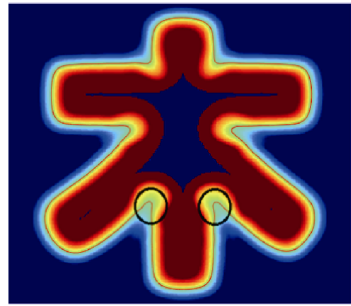

c. 迭代10次结果

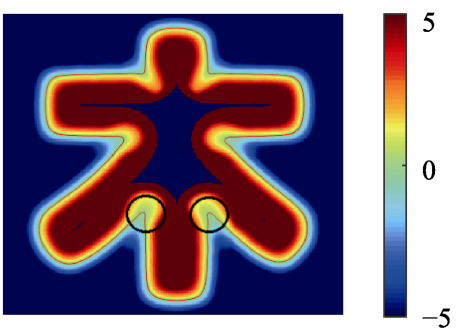

d. 迭代35次结果

图 3 汉字 “木”形状拟插值及隐式曲线几何迭代的重构结果

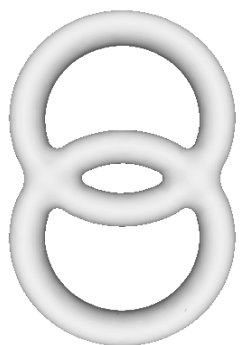

a. 原始采样点

b. 隐式几何迭代重构曲面

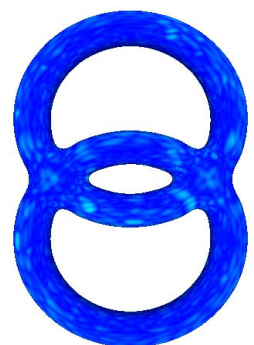

c. 迭代重构几何误差

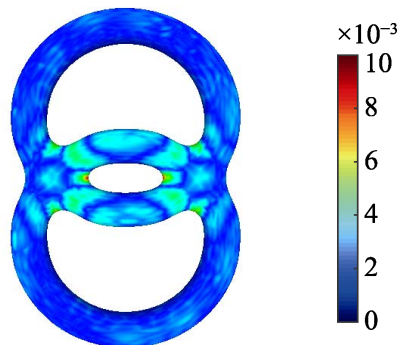

d. 拟插值结果 ${ }^{[6]}$ 几何误差

图 4 Double_Torus 模型曲面重构结果及其与拟插值几何误差

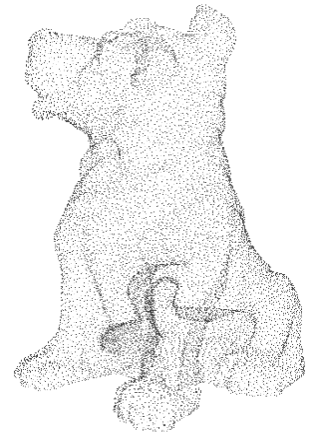

a. 原始采样点

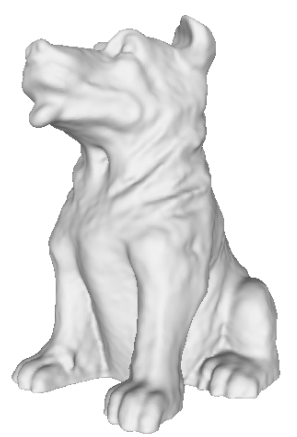

b. 隐式几何迭代重构曲面

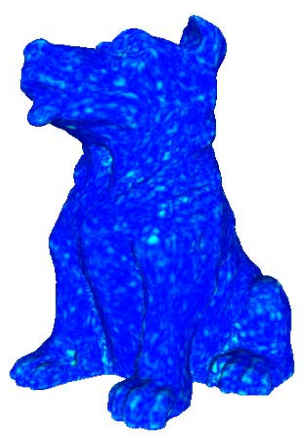

c. 迭代重构几何误差

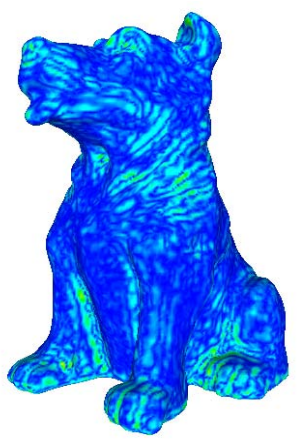

d. 拟插值结果 ${ }^{[6]}$ 几何误差

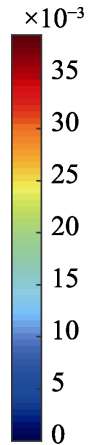

图 5 Dog 模型曲面重构结果及其与拟插值几何误差

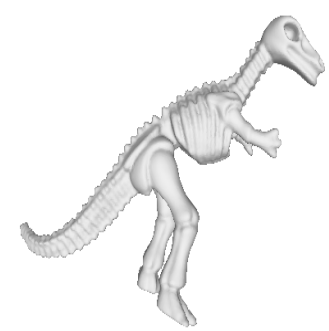

b. 隐式几何迭代重构曲面

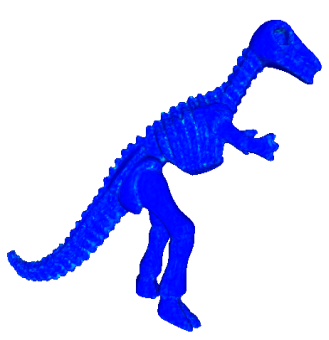

c. 迭代重构几何误差

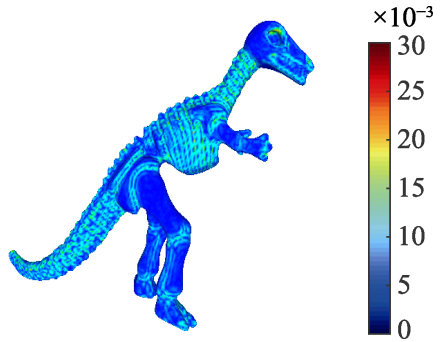

d. 拟插值结果 ${ }^{[6]}$ 几何误差

图 6 Dinosaur 模型曲面重构结果及其与拟插值几何误差

据点较少时，算法 2 在时间上也有优势. 但是在实 际计算中发现，在计算算法 2 的加速因子时, 需要 形成配置矩阵并计算其最小特征值. 当模型的数 据点增加时，计算的时间和空间复杂度也相应增 加, 如 Dog 模型等; 而对于顶点数目比较多的模
型，如 Dinosaur 模型，形成配置矩阵时由于计算机 内存不足而导致无法计算其最小特征值. 即使能 够形成配置矩阵，求矩阵最小特征值的过程会耗 时较长，导致尽管迭代次数较少，但实际整个算法 的运行时间比加速前更长. 为减少内存消耗, 以及 
避免耗时的配置矩阵最小特征值计算，使用配置 矩阵的主对角线最小元素代替其最小特征值. 将 算法 2 的改进版本标记为算法 $2+$.

由表 1 的统计数据, 算法 $2+$ 由于采用了最优 加速参数的估计值, 这将使加速算法收玫速度有 所下降, 迭代次数增加. 对于数据量较少的模型, 如 3 个二维的例子, 算法 $2+$ 与算法 2 的用时基本
相等，而Double_Torus 模型由于特征值的计算，导 致算法 2 比算法 $2+$ 耗时略长. 但依然比没有加速 的算法 1 迭代次数更少, 运行时间更短. 对于数据 量较大的模型，采用算法 $2+$ 避免了配置矩阵的构 造和其最小特征值的求解，降低了加速算法的空 间复杂度，且其重构时间也最短，真正起到了加速 的效果. 下文均采用算法 $2+$ 对模型进行处理.

表 1 算法有效性实验中算法 1 与算法 2 的结果统计

\begin{tabular}{|c|c|c|c|c|c|c|}
\hline 模型 & 数据点个数 & 支撑半径大小 & 算法比较项目 & 迭代时间/ms & 迭代次数 & 最大几何误差 \\
\hline \multirow{3}{*}{ 五角星 } & \multirow{3}{*}{132} & \multirow{3}{*}{3.0} & 算法 1 & 13 & 150 & \multirow{3}{*}{$2.24 \times 10^{-3}$} \\
\hline & & & 算法 2 & 6 & 75 & \\
\hline & & & 算法 2+ & 7 & 82 & \\
\hline \multirow{3}{*}{ 字母 G } & \multirow{3}{*}{193} & \multirow{3}{*}{3.0} & 算法 1 & 26 & 152 & \multirow{3}{*}{$1.89 \times 10^{-3}$} \\
\hline & & & 算法 2 & 15 & 76 & \\
\hline & & & 算法 2+ & 15 & 81 & \\
\hline \multirow{3}{*}{ 汉字“木” } & \multirow{3}{*}{248} & \multirow{3}{*}{2.0} & 算法 1 & 13 & 69 & \multirow{3}{*}{$2.48 \times 10^{-3}$} \\
\hline & & & 算法 2 & 7 & 35 & \\
\hline & & & 算法 2+ & 8 & 38 & \\
\hline \multirow{3}{*}{ Double_Torus } & \multirow{3}{*}{1474} & \multirow{3}{*}{3.0} & 算法 1 & 1274 & 208 & \multirow{3}{*}{$2.62 \times 10^{-3}$} \\
\hline & & & 算法 2 & 770 & 105 & \\
\hline & & & 算法 2+ & 713 & 108 & \\
\hline \multirow{3}{*}{ Dog } & \multirow{3}{*}{15000} & \multirow{3}{*}{1.0} & 算法 1 & 5352 & 181 & \multirow{3}{*}{$2.29 \times 10^{-3}$} \\
\hline & & & 算法 2 & 21077 & 91 & \\
\hline & & & 算法 2+ & 2818 & 94 & \\
\hline \multirow{3}{*}{ Dinosaur } & \multirow{3}{*}{56194} & \multirow{3}{*}{0.6} & 算法 1 & 153591 & 329 & \multirow{3}{*}{$2.65 \times 10^{-3}$} \\
\hline & & & 算法 2 & - & - & \\
\hline & & & 算法 2+ & 79514 & 168 & \\
\hline
\end{tabular}

注.一表示因内存不足，无法计算该模型的配置矩阵最小特征值.

\section{2 部分缺失采样曲面重构}

在实际工程中，经常会遇到通过设备扫描产 生的点云中出现缺失数据采样的情况, 如图 7 和图 8 所示. 如果采用文献[6]估计的支撑半径进行曲面 重构，会导致缺失数据采样的地方无法重构出正 确的曲面, 如图 $7 \mathrm{~b}$ 和图 $8 \mathrm{~b}$ 所示, 采用支撑半径分 别为 0.31 和 0.20 ; 如果分别使用如图 $7 \mathrm{c}$ 和图 $8 \mathrm{c}$ 所示比较大的支撑半径 0.8 和 0.6 , 尽管能把缺失 采样部分的曲面重构出来, 但是在细节丰富的地 方会丢失几何细节. 针对这一情况，利用本文算法 对部分缺失采样曲面进行重构, 从图 $7 \mathrm{~d}$ 和图 $8 \mathrm{~d}$ 中 可以看出，相比拟插值结果，细节部分得到了更好 的体现，可正确地重构采样缺失部分的曲面. 相关 统计数据如表 2 所示.

表 2 部分缺失采样曲面实验结果统计

\begin{tabular}{rccc}
\hline 模型 & 数据点个数 & 支撑半径 & 迭代时间 $/ \mathrm{s}$ \\
\hline Bimba & 72769 & 0.8 & 71 \\
$\mathrm{Bu}$ & 121651 & 0.6 & 139 \\
\hline
\end{tabular}

\section{3 非均匀采样曲面重构}

有很多点云模型为了压缩数据, 采用非均匀 采样的形式表示模型形状. 在细节丰富的地方数 据点比较稠密, 在曲面较为光滑的区域数据点稀 疏，或者由于数据获取过程操作不一致，导致模型 曲面的不同部分采样率不同. 如果依据整体点云 密度估计一个恰当的支撑半径, 然后采用单层拟 插值算法 ${ }^{[6]}$ 进行曲面重构, 会导致重构的曲面在采 样稀疏区域有洞，如图 $9 \mathrm{~b}$ 和图 $10 \mathrm{~b}$ 所示，其支撑半 径都为 0.23 . 如果根据稀疏区域采样密度估计一 个比较大的支撑半径，尽管会在稀疏区域重构出 连续的曲面, 但是在采样稠密区域的几何细节被 光滑掉, 如图 $9 \mathrm{c}$ 和图 $10 \mathrm{c}$ 所示, 支撑半径都为 0.8 . 本文算法使用这个相对较大的支撑半径, 经过迭 代后能由非均匀采样数据重构出高质量曲面, 既 能重构出光滑连续曲面, 又不丢失几何细节, 即使 在如图 9d 和图 10d 所示采样稀疏的区域. 重构结 果相关信息统计如表 3 所示. 


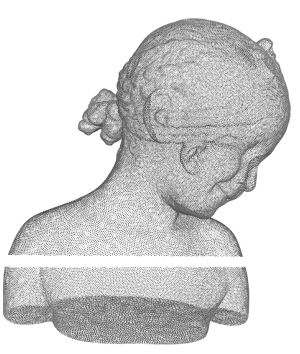

a. 原始采样点

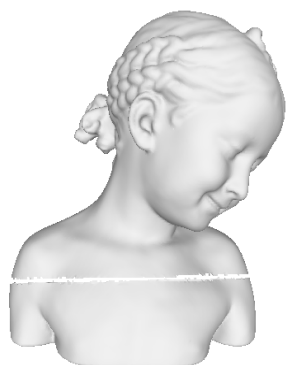

b. 支撑半径 0.31 拟插值结果 ${ }^{[6]}$

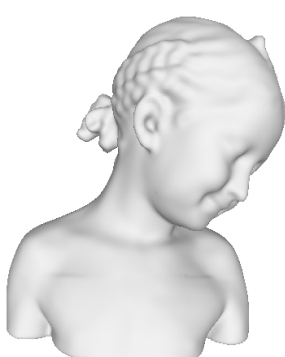

c. 支撑半径 0.8 拟插值结果 ${ }^{[6]}$

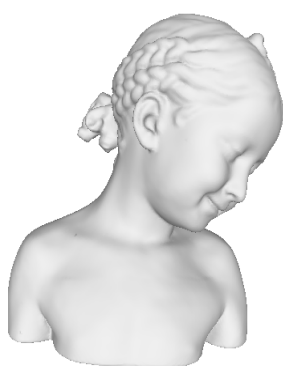

d. 迭代后结果

图 7 部分缺失采样 Bimba 模型拟插值以及隐式曲面几何迭代后的结果展示

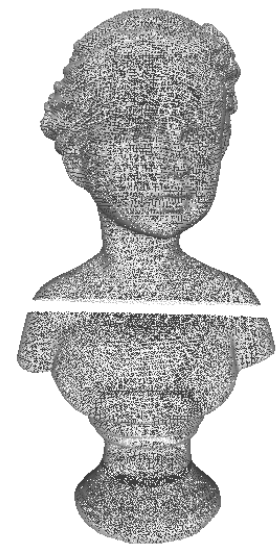

a. 原始采样点

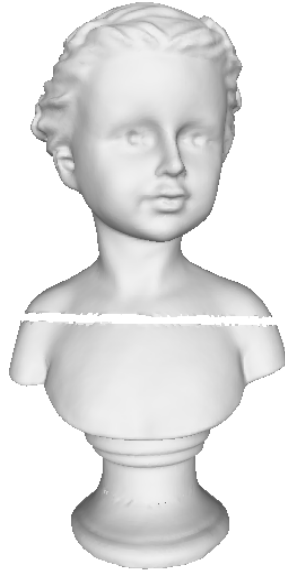

b. 支撑半径 0.20 拟插值结果 ${ }^{[6]}$

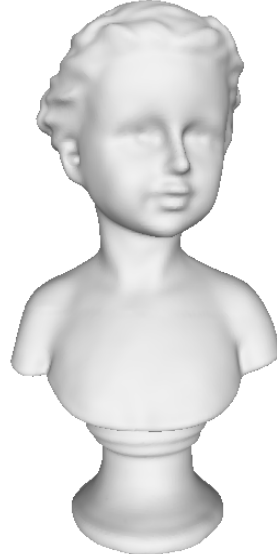

c. 支撑半径 0.6 拟插值结果 ${ }^{[6]}$

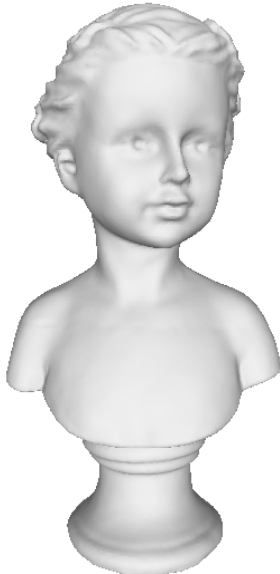

d. 迭代后结果

图 8 部分缺失采样 $\mathrm{Bu}$ 模型拟插值以及隐式曲面几何迭代后的结果展示

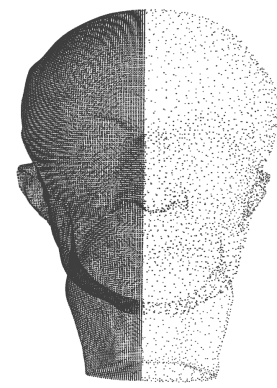

a. 原始采样点

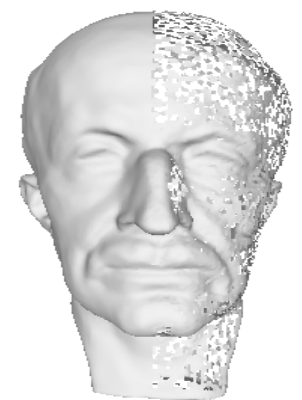

b. 支撑半径 0.23 拟插值结果 ${ }^{[6]}$

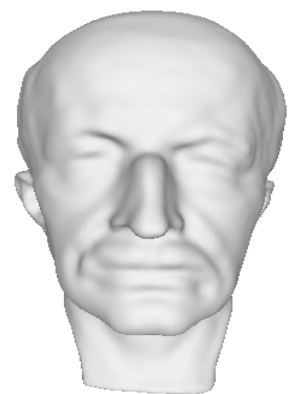

c. 支撑半径 0.8 拟插值结果 ${ }^{[6]}$

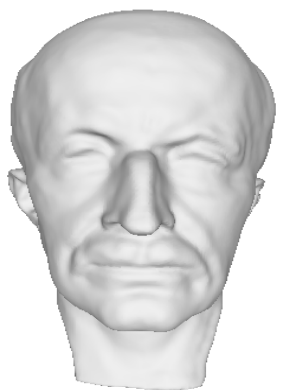

d. 迭代后结果

图 9 非均匀采样 MaxPlanck 模型拟插值以及隐式曲面几何迭代后的结果展示

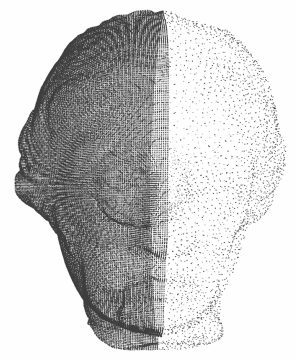

a. 原始采样点

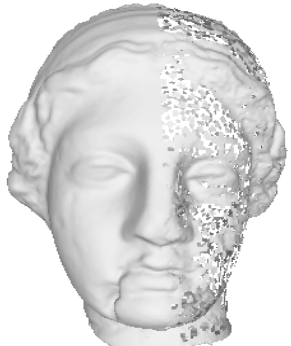

b. 支撑半径 0.23 拟插值结果 ${ }^{[6}$

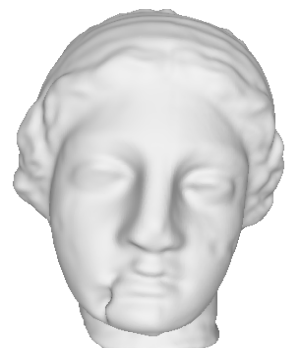

c. 支撑半径 0.8 拟插值结果 ${ }^{[6]}$

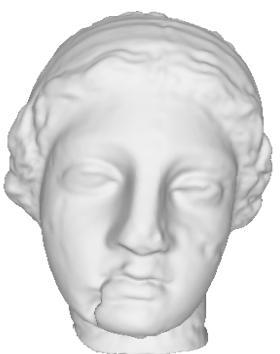

d. 迭代后结果

图 10 非均匀采样 Igea 模型拟插值以及隐式曲面几何迭代后的结果展示 
表 3 非均匀采样曲面实验结果统计

\begin{tabular}{lccc}
\hline \multicolumn{1}{c}{ 模型 } & 数据点个数 & 支撑半径 & 迭代时间 $/ \mathrm{s}$ \\
\hline MaxPlanck & 67369 & 0.8 & 111 \\
Igea & 100371 & 0.8 & 126 \\
\hline
\end{tabular}

\section{4 带噪声点云数据曲面重构}

本文算法还能处理带噪声的点云数据曲面重 构问题. 如果噪声比较大，则可以先使用文献[22] 方法对点云数据进行离群点去除, 然后通过正则 化 RBF 配置矩阵, 将 RBF 配置矩阵从插值转换为 逼近.

RBF 配置矩阵 $\boldsymbol{B}$ 可转换为正则矩阵 $\boldsymbol{B}+\tau \boldsymbol{I}$, 其中， $\tau$ 为正则化参数， $I$ 为单位矩阵. 当设置正 则化参数为 $\tau=n$ ( $n$ 为数据点个数) 时, 可以得到 较好的效果. 应用到迭代算法中，可以通过处理系 数解决, 用 $c_{i}^{(k)} /(1+\tau)$ 代替 $c_{i}^{(k)}$ 即可. 由于正则矩 阵严格对角占优, 收玫速度较快, 故重构结果与拟 插值对噪声数据的处理结果相似.

图 11a 展示了一个带噪声的具有 25 万个点的 Filigree 数据模型的处理实例, 图 $11 \mathrm{~b}$ 所示的曲面 重构结果是迭代 5 次的结果, 支撑半径为 0.6 , 重 构曲面的迭代过程用时 $62 \mathrm{~s}$.

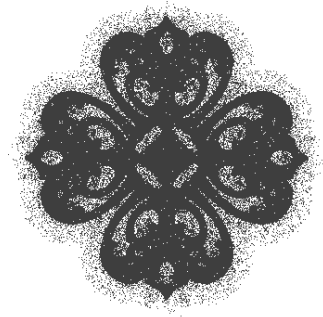

a. 原始采样点

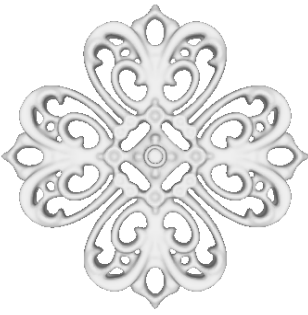

b. 迭代后结果
图 11 带噪声点云模型 Filigree 隐式曲面 几何迭代后的结果展示

\section{5 结 语}

在拟插值的基础上，本文提出了基于局部支 撑 RBF 的隐式曲线曲面几何迭代算法及其加速算 法, 并对 2 个算法的收玫性进行了讨论. 曲线曲面 重构数值实验验证了算法的有效性, 并针对加速 算法需要求解特征值的问题，提出了利用配置矩 阵对角线的最小元素替代最小特征值的办法，降 低了空间和时间复杂度，构成算法的一个改进版 本. 同时充分利用 $\mathrm{RBF}$ 处理散乱数据点的优势, 对非均匀采样数据、缺失采样数据、带噪声采样数 据进行曲面重构，弥补了因为支撑半径偏大而丢
失细节的缺陷, 达到了令人满意的效果. 此外, 本 文算法具有计算简便、易于并行的特点. 但是通过 实验发现，即使加速后，收玫速度仍有待提高，构 造更快的、具有明显几何意义的加速迭代格式是今 后需要进一步研究的方向.

\section{参考文献(References):}

[1] Dey T K. Curve and surface reconstruction: algorithms with mathematical analysis[M]. New York: Cambridge University Press, 2007: 173-202

[2] Wendland H. Scattered data approximation[M]. New York: Cambridge University Press, 2005

[3] Han X L, Hou M Z. Quasi-interpolation for data fitting by the radial basis functions[C] //Proceedings of the 5th International Conference on Advances in Geometric Modeling and Processing. Heidelberg: Springer, 2008: 541-547

[4] Liu S J, Wang C C L. Quasi-interpolation for surface reconstruction from scattered data with radial basis function[J]. Computer Aided Geometric Design, 2012, 29(7): 435-447

[5] Pan R J, Meng X X, Whangbo T K. Hermite variational implicit surface reconstruction[J]. Science in China: Series F, 2009, 52(2): 308-315

[6] Liu S J, Brunnett G, Wang J. Multi-level Hermite variational interpolation and quasi-interpolation[J]. The Visual Computer, 2013, 29(6-8): 627-637

[7] Qi Dongxu, Tian Zixian, Zhang Yuxin, et al. The method of numeric polish in curve fitting[J]. Acta Mathematica Sinica, 1975, 18(3): 173-184(in Chinese)

(齐东旭, 田自贤, 张玉心, 等. 曲线拟合的数值磨光方 法 $[\mathrm{J}]$. 数学学报, $1975,18(3): 173-184)$

[8] de Boor C. How does Agee's smoothing method work?[R]. Washington D C: Army Research Office, 1979: 299-302

[9] Lin H W, Wang G J, Dong C S. Constructing iterative non-uniform B-spline curve and surface to fit data points[J]. Science in China: Series F, 2004, 47(3): 315-331

[10] Lin H W, Bao H J, Wang G J. Totally positive bases and progressive iteration approximation[J]. Computers \& Mathematics with Applications, 2005, 50(3/4): 575-586

[11] Lin H W. Local progressive-iterative approximation format for blending curves and patches[J]. Computer Aided Geometric Design, 2010, 27(4): 322-339

[12] Lin H W, Zhang Z Y. An extended iterative format for the progressive-iteration approximation[J]. Computers \& Graphics, 2011, 35(5): 967-975

[13] Deng C Y, Lin H W. Progressive and iterative approximation for least squares B-spline curve and surface fitting[J]. Computer-Aided Design, 2014, 47: 32-44

[14] Lin H W, Cao Q, Zhang X T. The convergence of least-squares progressive iterative approximation for singular least-squares fitting system[J]. Journal of Systems Science and Complexity, 2018, 31(6): 1618-1632

[15] $\mathrm{Lu} \mathrm{L} \mathrm{Z.} \mathrm{Weighted} \mathrm{progressive} \mathrm{iteration} \mathrm{approximation} \mathrm{and}$ convergence analysis[J]. Computer Aided Geometric Design, 2010, 27(2): 129-137 
[16] Zhang Li, Zhao Lin, Tan Jieqing. Progressive iterative approximation with different weights and its application[J]. Journal of Zhejiang University: Science Edition, 2017, 44(1): 22-27(in Chinese)

(张莉，赵林，檀结庆。带互异权值的渐进迭代逼近算 法及其应用 [J]. 浙江大学学报：理学版，2017，44(1): 22-27)

[17] Hamza Y F, Lin H W, Li Z H. Implicit progressive-iterative approximation for curve and surface reconstruction[J]. Computer Aided Geometric Design, 2020, 77: 101817

[18] Han Xuli. Numerical analysis[M]. Changsha: Central South University Press, 2003(in Chinese)

(韩旭里. 数值分析 [M]. 长沙: 中南大学出版社, 2003)

[19] Tang Jianguo. Upper and lower bound of eigenvalues for multiplication of two real symmetric matrices[J]. Journal of Yanbian University: Natural Science, 2009, 35(4): 302-304(in Chinese)

(唐建国. 两实对称矩阵乘积特征值的上下界 $[\mathrm{J}]$. 延边 大学学报：自然科学版, 2009, 35(4): 302-304)

[20] Ohtake Y, Belyaev A G, Alexa M, et al. Multi-level partition of unity implicits[J]. ACM Transactions on Graphics, 2003, 22(3): 463-470

[21] Kazhdan M, Hoppe H. Screened Poisson surface reconstruction[J]. ACM Transactions on Graphics, 2013, 32(3): Article No.29

[22] Liu S J, Chan K C, Wang C C L. Iterative consolidation of unorganized point clouds[J]. IEEE Computer Graphics and Applications, 2012, 32(3): 70-83 